\title{
La difusión de la idea de migrar. Andalucía y América en el tránsito del siglo XIX al XX
}

\author{
Francisco Contreras Pérez \\ Departamento de Historia II \\ Universidad de Huelva
}

Teniendo en cuenta la importancia de la masiva emigración contemporánea de Andalucía a América, que presenta nuevos componentes respecto a la de época colonial, se ensaya la integración de las ocho corrientes migratorias provinciales en un esquema explicativo de los procesos de difusión de la idea de emigrar entre la población andaluza. Para su realización, sometemos a examen y aplicación el modelo defendido por J. D. Gould, especialmente adecuado al uso de fuentes estadísticas agregadas (compatibles con las usadas por nosotros) y aplicado para explicar otras corrientes migratorias regionales europeas. Este estudio crítico del modelo sometido a examen tiene la virtualidad de permitir establecer hipótesis de trabajo preliminares y necesarias, que pretendemos poner a prueba mediante el empleo de fuentes microhistóricas (como las listas de pasajeros o partes consulares).

En este ensayo, ${ }^{1}$ que abrimos con una breve aproximación a la evolución de conjunto de la emigración en masa de andaluces entre los siglos XIX y $\mathrm{XX}$, pretendemos poner a prueba un esquema explicativo de los procesos de

1 Las principales fuentes primarias que hemos consultado corresponden a las elaboradas por el Instituto Geográfico y Estadístico, pudiéndose consultar en las Delegaciones del Instituto Nacional de Estadísitica de Huelva y Sevilla (microfichas de los censos) y la biblioteca del Ministerio de Trabajo (boletines de migraciones): Censo de la población de España según el recuento verificado en 24 de mayo de 1857 (Comisión de Estadística General del Reino), Madrid, 1858; Censo de la población de España según el recuento verificado en 25 de diciembre de 1860, Madrid, 1863; Censo de la población de España según el empadronamiento hecho en 31 de diciembre de 1877, Madrid, 1883; Censo de la población de España según el empadronamiento hecho en 31 de diciembre de 1887, Madrid, 1891; Resultados provisionales del censo de la población de España según el empadronamiento hecho en la Península e islas adyacentes el 31 de diciembre de 1897, Madrid, 1899; Censo de la población de España según el empadronamiento hecho en la Península e islas adyacentes el 31 de diciembre de 1900, Madrid, 1902; Censo de la población de España según el empadronamiento hecho en la Península e islas adyacentes en 31 de diciembre de 1910, Madrid, 1913; Censo de la población de España según el empadronamiento hecho en la Península e islas adyacentes el 31 de diciembre de 1920, Madrid, 1922; Censo de la población de España según el empadronamiento hecho en la Península e islas adyacentes del norte y costa occidental de África el 31 de diciembre de 1930, Madrid, 1932; Estadística de la emigración e inmigración de España en los años de 1882 a 1890, Madrid, 1891; Estadística de la emigración e inmigración de España en el quinquenio 1891-1895, Madrid, 1898; Estadística de la emigración e inmigración de España en el quinquenio de 1896-1900, Madrid, 1903; Estadística de la emigración e inmigración de España en los años 1901 y 1902, Madrid, 1903; Estadística de la emigración e inmigración de España en los años 1903 a 1906, Madrid, 1907; Estadística de la emigración e inmigración de España en los años 1907 y 1908, Madrid, 1910; Estadística de la emigración e inmigración de España 
difusión de la idea de migrar integrando las distintas corrientes provinciales. Sin menoscabo de que el referente del indiano fuera un viejo topo en el imaginario popular, hay que hacer constar que dichos procesos de divulgación, en el marco de la modernización socioeconómica de Europa, se asemejan a las pautas seguidas por la popularización de las numerosas innovaciones tecnológicas y socioculturales que empiezan a caracterizar el nuevo siglo XX (el uso de la máquina de coser, por ejemplo). Por otro lado, aún reconociendo las limitaciones analíticas de la escala provincial (precisadas más adelante), ésta tiene la virtualidad de permitir el afianzamiento de hipótesis de trabajo, imprescindibles a la hora de abordar, contrastar y evaluar con exactitud los ulteriores estudios microhistóricos que estamos llevando a cabo.

Desde un punto de vista metodológico, se ha dicho que la decisión de migrar puede ser simplificada en tres elementos interrelacionados. En primer lugar, la resolución de elegir entre los actos de migrar y permanecer. A continuación, la determinación de migrar sólo podría ser abordada después de haber recibido noticias adicionales sobre uno o varios factores (empleo, salarios, acceso a la propiedad, etc., en los lugares alternativos). Por último, actos y factores tienen una consecuencia para el que toma dicha resolución. ${ }^{2}$ Así pues, la información constituye una pieza clave en el proceso migratorio, entendido en definitiva como un sistema secuencial de toma de decisión. De hecho, en su tesis sobre los determinantes de la emigración española, B. Sánchez Alonso, tras evaluar la relación entre emigración y una serie de factores explicativos (socioeconómicos y niveles de alfabetismo), estima que existe una correlación positiva y significativa con esta última variable, por lo que concluye: "se confirma plenamente para el caso español el papel crucial que el acceso a la información desempeña en el proceso emigratorio, tanto en el siglo XIX como en el XX". ${ }^{3}$

en los años 1909, 1910 y 1911, Madrid, 1912; Estadística de pasajeros por mar. Años 1912 y 1913, Madrid, 1914; Estadística de pasajeros por mar. Año 1914, Madrid, 1916; Estadística de pasajeros por mar. Año 1915, Madrid, 1917; Estadística de pasajeros por mar. Año 1916, Madrid, 1917; Estadística de pasajeros por mar. Años 1917 y 1918, Madrid, 1922; Estadística de pasajeros por mar. Año 1919, Madrid, 1923; Estadística de pasajeros por mar. Años 1920-1921-1922, Madrid, 1924; Estadística del movimiento de buques y pasajeros por mar con el exterior. Años 1923, 1924 y 1925, Madrid, 1929; Estadística del movimiento de buques y pasajeros por mar con el exterior. Años 1926, 1927, 1928 y 1929, Madrid, 1932; Estadística del movimiento de buques y pasajeros por mar con el exterior. Año 1930, Madrid, 1933.

2 Lewis, G.J.: Human Migration. A Geographical Perspective, Londres, 1982, pág. 127.

3 Sánchez Alonso, B.: Las causas de la emigración española 1880-1930, Madrid, 1995, págs. 203-270 y 278. El papel de los procesos de difusión cobra cada vez más valor explicativo en diferentes ámbitos de estudio, como ha puesto de relieve M. Livi Bacci acerca de las pautas regionales en la transición demográfica española. Este autor denomina "factor regional" a una serie de variables imponde- 
El procedimiento por el que un individuo acumula información sobre la alternativa, está guiado por la amplitud y contenido de su campo de información, o el conjunto de lugares sobre los que tiene conocimiento. Tal campo puede estar dividido en dos: un espacio de actividad y un espacio de contacto indirecto. ${ }^{4}$ En el marco de las migraciones transoceánicas, el contacto indirecto era obviamente el principal canal de información (a excepción de las migraciones golondrinas, frecuentes entre canarios a América $^{5}$ y almerienses a Argelia, por ejemplo). Además, el proceso de toma de decisión de los candidatos a emigrar no sólo depende del conocimiento individual o colectivo de las oportunidades alternativas, sino también de los tipos de información disponible. ${ }^{6}$ En las migraciones masivas de Europa a América, podemos considerar dos tipos básicos de canales de información: ${ }^{7}$ por un lado, amigos y parientes y, por otro, los agentes de emigración (representantes consulares americanos, agentes y consignatarios de compañías navieras, padroni, etc.). ${ }^{8}$ Con funcionamientos distintos, ${ }^{9}$ estos dos canales contribuyeron a difundir el ansia por migrar, y a posibilitarla en sus distintas formas. Pero antes de analizar su operatividad, se hace imprescindible hacer un seguimiento, aunque sea breve, de la evolución de la emigración andaluza en su conjunto.

rables, diferentes de los factores económicos tradicionalmente aducidos y de difícil individualización, que nos remiten en última instancia a un espacio histórico y cultural homogéneo en el que los nuevos comportamientos se habrían difundido más fácilmente. Livi Bacci, M.: "La Península Ibérica e Italia en vísperas de la transición demográfica”, en Pérez Moreda, V. y Reher, D.-S.: Demografía histórica en España, Madrid, 1988, págs. 138-178.

4 Lewis: Human Migration..., pág. 142.

5 Macías, A. M.: "Un siglo de emigración canaria”, en Sánchez-Albornoz, N. (comp.): Españoles hacia América. La emigración en masa, 1880-1930, Madrid, 1988, págs. 166-202.

6 Lewis: Human Migration..., pág. 146.

7 Akerman, S.: "Towards an Understanding of Emigrational Processes", en McNeil, W. y Adams, R. (eds.): Human Migration. Patterns and Policies, Indiannapolis, 1978, págs. 295-300.

8 El sistema del padrone o boss, de fundamental importancia en la contratación de trabajadores italianos con destino a Estados Unidos, es descrito en Avagliano, L.: L'emigrazione italiana, Nápoles, 1976, págs. 121-129. Para el caso de Andalucía, hemos analizado el funcionamiento de las agencias de emigración americanas y sus delegados en "Recluta masiva de emigrantes andaluces y su inserción social en Argentina (siglo XIX). Nuevas notas para su estudio", Anuario de Estudios Americanos, t. LIII, n. ${ }^{\circ}$ 2, Sevilla, 1996, págs. 173-197, en "El río revuelto de la emigración: el papel de las agencias gibraltareñas a principios de siglo", Almoraima. Revista de estudios campogibraltareños, n.․16, Algeciras, 1996, págs. 63-73, y en "El río revuelto de la emigración (II): el papel de las navieras y los estados en Gibraltar a principios de siglo", Almoraima. Revista de estudios campogibraltareños, n. ${ }^{\circ}$ 18, Algeciras, 1997, págs. 71-78.

9 Para una síntesis genérica del funcionamiento de las redes de reclutamiento y de las cadenas migratorias o redes sociales, véase Vázquez, A. y Estrada, B.: "Causas de la emigración y tipología de los emigrantes", en Historia General de la emigración española a América, Madrid, 1992, t. 1, págs. 201-240. 


\section{Una evaluación preliminar de la emigración andaluza}

Como precedente obligado en la historiografía andaluza, el trabajo de Mateo Avilés supone un primer acercamiento al análisis del "volumen y la periodificación" de la emigración andaluza hacia América. Como conclusión acerca de este punto, dicho autor estima que, sin ser el más numeroso de Europa, "el éxodo andaluz resulta, en volumen global [según algunas aproximaciones teóricas, unos 500.000 emigrantes], significativo e importante en torno al 15\% dentro de la aportación española a ese grandioso movimiento de población" trasatlántico. ${ }^{10}$

Dicho lo cual, pretendemos precisar, en la medida de lo posible, este análisis con un enfoque longitudinal que permita observar las fluctuaciones de la emigración andaluza hacia el exterior en su conjunto, desde una perspectiva comparativa ${ }^{11}$ y mediante el empleo de indicadores homogéneos, ${ }^{12}$ como instrumentos adecuados para aproximarnos de manera más rigurosa a los sucesivos niveles emigratorios presentados por la región. Con este proceder, pretendemos discernir entre lo común y lo específico de la tendencia y fluctuaciones trazadas por el flujo exterior andaluz.

A largo plazo, la evolución de la emigración andaluza presenta la conocida forma de curva en S (véase la fig. 1), que caracteriza generalmente el perfil longitudinal del masivo ciclo migratorio europeo desde fines del siglo XIX a principios del XX. ${ }^{13}$

En la etapa estadística del siglo XIX, la primera gran oleada emigratoria andaluza tiene lugar en los años finales de la década de 1880, siendo

10 Mateo Avilés, E. de: La emigración andaluza a América (1850-1936), Málaga, 1993, págs. 113-127.

11 Green, N., "L'histoire comparative et le champ des études migratoires", Annales. Economie, Société, Civilization, n. ${ }^{\circ}$ 6, París, 1990, págs. 1335-1350. La autora, estudiosa de la inmigración en Francia, hace constar que "la comparación nos parece esencial como camino para poder evaluar lo que se deriva del conjunto y lo que pertenece a lo específico" (traducción personal).

12 Según estos parámetros relativos, intensidad y peso proporcional emigratorios de los flujos regionales, se pueden agrupar las regiones en tres tipos: 1) Regiones con escasa participación en la emigración española: el porcentaje con que participan en la emigración española es menor al de la población española que representan, y su tasa de emigración bruta por cada mil habitantes es siempre menor a la tasa española del período. 2) Regiones con niveles intermedios de emigración: porcentajes de participación emigratoria aproximados al peso de su población, y tasas de emigración también próximas a la media española. 3) Regiones con altos niveles migratorios: porcentajes de emigración superiores al peso de su población en el total español, y tasas de emigración bruta mayores al nivel medio de España. Yáñez Gallardo, C.: La emigración española a América (ss. XIX y XX). Dimensión y características cuantitativas, Colombres, págs. 235-241.

13 Gould, J. D.: 'European Inter-Continental Emigration: The Role of 'Diffusion' and 'Feedback'”, en The Journal of European Economic History, n. ${ }^{2}$, Londres, 1980, págs. 267-315. 
el de máxima emigración 1889. Por el contrario, toda la década de 1890 es de descenso, aunque con pequeñas fluctuaciones. Reactivada a principios de siglo, la emigración andaluza alcanza un nuevo máximo en 1912. Máximo que fue en realidad el momento de apogeo emigratorio, teniendo en cuenta que este nuevo flujo regional está infravalorado en mayor medida a raíz de que, entrada en vigor la Ley de 1907, las agencias reclutadoras desviaran con mayor frecuencia los embarques hacia el puerto de Gibraltar. A partir de la primera guerra mundial se retoma la tendencia a la caída que, después de un fuerte rebrote en 1920, se hizo clara en 1923-24 y se acentuaría en la década de 1930, cuando se cierra este ciclo migratorio en el mundo atlántico.

FIG. 1.-EMIGRACIÓN EXTERIOR.

ANDALUCÍA, GALICIA Y ESPAÑA, 1885-1930

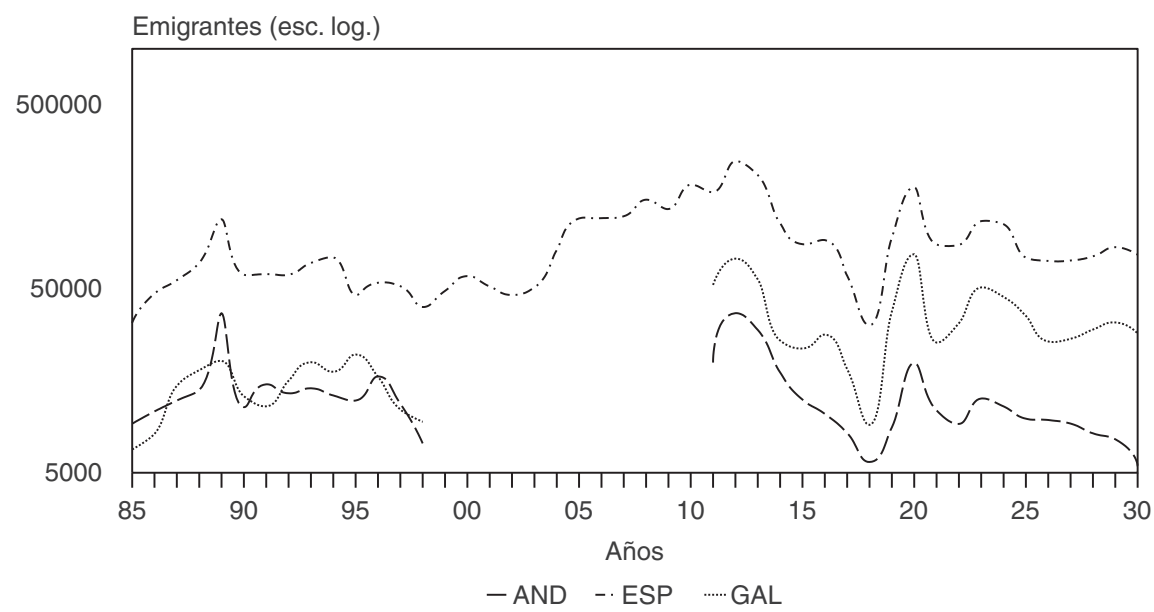

Fuente: Instituto Geográfico y Estadístico (IGE) (varios años).

La comparación de estas tendencias de la curva de emigración bruta andaluza con las de España y Galicia, región emigratoria por excelencia, ${ }^{14}$ resulta significativa. A simple vista se observan similitudes, pero también discrepancias (véase la fig. 1). Existen tres momentos de coincidencia entre

14 Juana, J. de: V Jornadas de Historia de Galicia. Galicia y América: el papel de la emigración, Orense, 1990. 
las series: la orientación claramente ascendente de los años 1885-89, más tardía y acusada en el caso andaluz; una segunda tendencia alcista en los años 1911-1913, cuando España y Andalucía alcanzan sus mayores máximos emigratorios; por último, en el período 1914-19, una profunda crisis durante la primera guerra mundial, seguida por un fuerte rebrote en 1920, que fue especialmente acelerado en el caso gallego y más moderado en el caso andaluz.

Las mayores discrepancias se producen a causa de la gran aceleración de la emigración andaluza en 1889, y a su brusco descenso posterior, incluso más acusado que en el caso español y, desde luego, opuesto a la tendencia ascendente de la emigración gallega en 1891-95. La emigración andaluza se mantiene, excepto en 1889 y 1891, por debajo de los niveles migratorios de la emigración gallega.

Así pues, lo que resulta específico del caso andaluz no sería tanto que se produjera un incremento de la emigración en la década de 1880, sino que esa subida fuera más pronunciada y, a la postre, menos sostenida que en los conjuntos español y gallego. Del mismo modo, se podría señalar que la aceleración de la emigración andaluza en los primeros años del siglo XX fue menor que en España y Galicia, como se observa comparando las tasas de crecimiento anual acumulativo de la emigración en las tres unidades geográficas (véase la tabla 1).

\section{TABLA 1}

EMIGRACIÓN EXTERIOR.

TASAS DE CRECIMIENTO MEDIO ANUAL, 1885-1930 (× 100)

Región / país $\quad$ 1885-1912 1885 -1889 $1885-1898$ 1898-1912 $1920-1930$

\begin{tabular}{lrrrrr}
\hline Andalucía & 5,25 & 41,23 & $-1,87$ & 7,47 & $-12,09$ \\
Galicia & 7,90 & 31,86 & 2,71 & 15,65 & $-9,43$ \\
España & 7,77 & 38,22 & 1,53 & 13,90 & $-8,10$
\end{tabular}

Fuente: Véase nota 1. Elaboración personal.

En el conjunto de este período clásico de la emigración transoceánica, 1885-1912, Andalucía presentó la tasa de crecimiento menos elevada. Las mayores discrepancias sin embargo se observan en los períodos correspondientes a los siglos XIX y XX respectivamente. Andalucía alcanzó la 
mayor aceleración migratoria en 1885-1889, superior a la de otras regiones migratorias. Pero en la década de los 90 la desaceleración resultó también más acusada. La subida de principios del siglo XX fue, por otra parte, bastante más moderada, y estuvo seguida de la más fuerte caída al final del período de emigración en masa.

Aun suavizando las fluctuaciones de estas series temporales, las líneas de tendencia siguen mostrando discrepancias significativas entre los comportamientos migratorios de Andalucía y Galicia en los períodos considerados: 1885-1895 y 1911-1930 (figs. 2 y 3). La emigración andaluza presenta en ambos períodos una tendencia descendente, si bien resulta más acusada en 1911-30. Esto indica que no hubo en los años inmediatos un flujo migratorio sostenido de entidad tras el "aluvión" de 1889. Por el contrario, la serie gallega presenta una línea ascendente en el primer período, resultado de un fuerte y conocido proceso de retroalimentación de la corriente migratoria. A su vez, en 1911-30 la pendiente de la emigración gallega, siempre presentando niveles migratorios superiores, es ligeramente menos acusada que el declive trazado por la emigración andaluza. El resultado final del conjunto del ciclo 1885-1930 es una línea integrada de tendencia ascendente en la emigración gallega y descendente en la andaluza.

\section{Los canales de difusión de la idea de migrar}

Los agentes de emigración y los amigos/parientes contribuyeron a difundir las ventajas de la emigración y a posibilitarla en sus distintas formas. Su acción de información, estímulo y ayuda financiera determinó tanto la decisión de migrar como la de hacia dónde migrar, ${ }^{15}$ entre otros aspectos.

\section{Las agencias de emigración}

Este mecanismo de emigración estuvo constituido por redes de agentes (consulares y de compañías navieras) y subagentes (ganchos) que, trabajando en las regiones emisoras, intervinieron en el proceso de configuración de

15 La distinción entre la decisión de trasladarse y la decisión de hacia dónde trasladarse, fue introducida por Brown y Moore al entender la migración como resultado de una "toma secuencial de decisión residencial". No obstante las deficiencias criticadas en el modelo de migraciones urbanas de Brown y Moore, consideramos útil y obvia esta distinción. Para una síntesis crítica de los trabajos de estos investigadores norteamericanos, véase Lewis: Human migration..., págs. 129-133. 
FIG. 2.-EMIGRACIÓN EXTERIOR.

ANDALUCÍA Y GALICIA, 1885-1898.

Líneas de tendencia

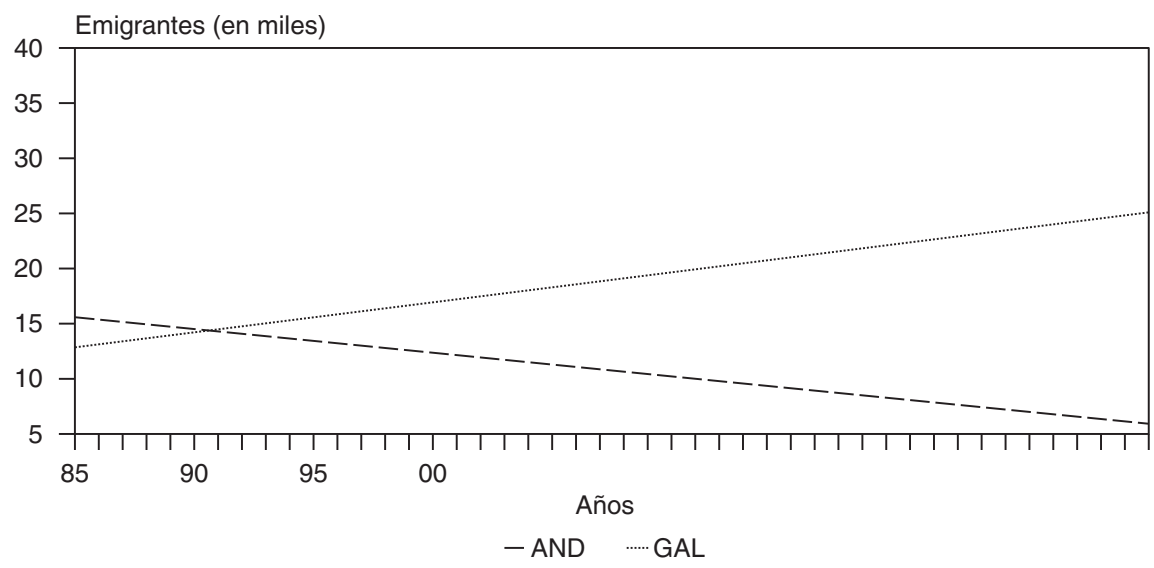

Fuente: IGE (varios años).

FIG. 3.-EMIGRACIÓN EXTERIOR.

ANDALUCÍA Y GALICIA, 1911-1930.

Líneas de tendencia

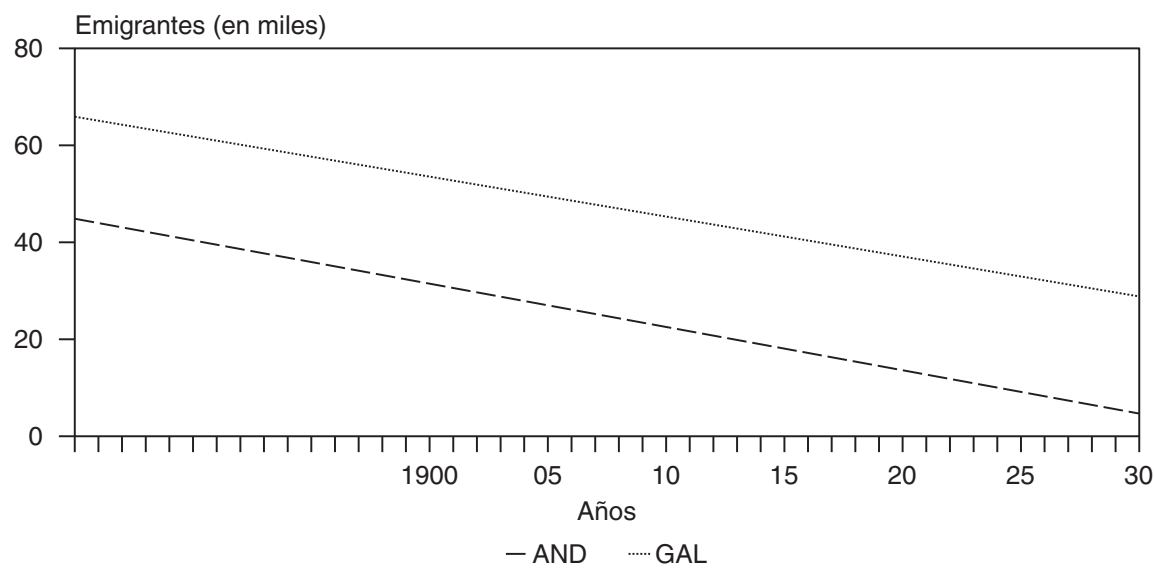

Fuente: IGE (varios años). 
la toma de decisión de los candidatos a migrar. Su labor estuvo íntimamente vinculada a los proyectos de pasajes subvencionados por empresas y, principalmente, gobiernos americanos. Entre éstos, los gobiernos de Argentina y Brasil fueron los más activos a partir de la década de los ochenta del siglo XIX. La atracción de colonos europeos se consideraba fundamental para poner en explotación y bajo dominio efectivo del Estado sus enormes territorios, y, en general, para modernizar las nuevas repúblicas.

La concreción de la "utopía agrícola americana" trajo consigo la nueva función propagandista de los representantes consulares hispanoamericanos, y la aparición nutrida de agencias de emigración, catapultas hacia el Cono Sur de los apetecidos colonos del Viejo Mundo. ${ }^{16}$

Los protagonistas de estas agencias, los agentes y ganchos, cumplieron a la perfección su trabajo, no tanto por filantropía o idealismo, sino por tratarse de una oportunidad de negocio. Su trabajo era a comisión, obtenida de las compañías navieras y de los gobiernos a los cuales estaban vinculadas. Los sistemas de embarque y contratación de inmigrantes daban mucho margen para especular, debido a las importantes cantidades que traían consigo las operaciones mercantiles relacionadas con la emigración. Sobra decir que sus métodos y formas de actuación no siempre respondían a normas morales o legales, sino que también para ellos los "negocios son los negocios".

Numerosos ya a mediados del siglo XIX en regiones de "vieja emigración", los centros de enganche estaban situados en los puertos. Desde allí, tendían sus radios de acción hacia las zonas del interior por medio de una red de subagentes que efectuaban los primeros contactos con los "candidatos" a la emigración. La superpoblación, las malas cosechas, las plagas y las sequías posibilitaban sus discursos oportunistas que, muchas veces, abrían alternativas al desaliento del campesinado. Esta labor de propaganda - casi nunca castigada - se completaba con la circulación de folletos, carteles, anuncios en prensa,... Todo ello, claro está, siempre exagerado, proporcionando en general al lector o al oyente una falsa imagen del trabajo y la riqueza en América. ${ }^{17}$ En Europa, las agencias de las compañías de vapores fueron acusadas del desarraigo de centenares de millares de habitantes en diferentes países. Si bien S. Akerman prefiere relativizar la labor

16 Azcona, J. M.: "El poder de los ganchos. El negocio de las agencias vascas de emigración en el siglo XIX", comunicación al IV Encuentro de Americanistas (9-11 de noviembre de 1994), Fundación Sánchez Albornoz, Oviedo, pág. 1-13 (inédito).

17 Ibídem, págs. 1-13. 
de estos agentes afirmando que, más que incidir en la oleada de emigración, se aprovecharon de ella. ${ }^{18}$

Uno de los mecanismos más utilizados por los gobiernos americanos para atraer migrantes fue encargar esta misión a sus funcionarios consulares. A pesar de la polémica suscitada en Argentina por los partidarios de las virtudes de la "migración espontánea", esto fue así, sobre todo, a partir de la década de los ochenta, años en los que se abren "oficinas de información para emigrantes" en las principales capitales europeas. ${ }^{19}$ Por último, la decidida política de anticipo de pasajes a los emigrantes por los gobiernos americanos (Brasil primero, después Argentina) desde 1888, se tornó un decisivo estímulo complementario de las acciones propagandísticas de los agentes. Sólo desde entonces se alcanzaron niveles realmente masivos de emigración hacia estos países en Andalucía y en el conjunto español.

\section{Cadenas migratorias y redes sociales ${ }^{20}$}

Otro de los más significativos canales es el que está formado a nivel del grupo primario de amigos y parientes. Denominando a este proceso en primera instancia "cadena migratoria", posteriormente también "red social" (ambos tan interrelacionados como para justificar su uso indistinto en la literatura científica), ${ }^{21}$ fue la pareja de investigadores americanos compuesta por John S. y Leatrice D. Macdonald quienes le dieron una ya clásica definición: "cadena migratoria puede ser definida como ese movimiento por el que los migrantes potenciales conocen de las alternativas, son provistos de transpor-

18 Akerman: "Towards an Understanding...”, págs. 295-300.

19 Vázquez, A. y Estrada, B.: “Causas de la emigración...”, págs. 201-240.

20 Como adelantamos, el concepto "cadenas migratorias" (o "redes sociales") hace referencia a los procesos migratorios que se apoyan en los mecanismos interpersonales e informales de información, ayuda financiera y acogida establecidos entre una comunidad en origen y otra en destino. Desde que en 1951 R. A. Lachore publicó su estudio From Europe to New Zealand, este concepto ha sido para los estudiosos una importante herramienta de investigación. Uno de los que más uso han hecho de este instrumento de investigación es Samuel L. Baily, que ofrece unas notas de reflexión sobre el concepto de "cadenas migratorias" o "redes sociales", y los interrogantes aún sin respuesta al respecto, en "Cadenas migratorias de italianos a la Argentina: algunos comentarios", Estudios migratorios latinoamericanos, n. ${ }^{\circ} 8$, Buenos Aires, 1988, págs. 125-135. Baily adopta igualmente una perspectiva comparativa en el estudio de las cadenas migratorias en "The Adjustment of Italian Inmigrants in Buenos Aires and New York, 1870-1914", en The American Historical Review, vol. 88, n. ${ }^{\circ}$ 2, Nueva York, 1983, págs. 281-305. Estos enfoques microhistóricos configuran las líneas de investigación entre los estudioso argentinos, con el Centro de Estudios Migratorios Latinoamericanos al frente.

21 A este respecto, es un buen ejemplo el estudio de Yáñez Gallardo, C.: Saltar con red. La temprana emigración catalana a América, 1830-1870, Madrid, 1996. 
te y obtienen acomodo y empleo a través, principalmente, de su contacto con migrantes previos", ya sean estos parientes, amigos o vecinos. ${ }^{22}$

Los migrantes previos (first comers) ${ }^{23}$ no sólo proporcionan información personalizada acerca de las oportunidades (a veces positivamente exageradas) del país receptor, sino que también pueden asistir a la financiación (billetes de llamada) y asimilación del recién llegado. Se constituyen así los sucesivos eslabones del mecanismo "cadena migratoria" y/o "red social".

Como es fácil de comprender, este conjunto de contactos personales, ${ }^{24}$ que forma la cadena migratoria, posee elementos de "realimentación", ${ }^{25}$ pues tiende a crear y sostener una corriente migratoria en el tiempo y a dirigirla en el espacio. Esta corriente llega a unir dos lugares o áreas separadas, el destino y el origen, mediante líneas de transmisión informativa y flujos financieros a escala familiar. ${ }^{26}$ Ya para la época colonial, se ha constatado este tipo de relaciones interpersonales mediando las aún mayores distancias atlánticas (la introducción del vapor en los barcos las reduciría en el siglo XIX, estrechando el mundo) gracias a las cartas y las terceras personas. ${ }^{27}$.

Mientras en origen las cadenas migratorias parecen tener un efecto de cascada en la difusión de la idea de emigrar (casi en progresión geométrica), en destino tiende a generar una concentración "étnica" o por "naturaleza" (lugar de nacimiento) de los migrantes. Así pues, y dado que el campo de información, o conjunto de lugares de destino conocidos mediante la cadena migratoria, era de hecho limitado, se dio en América una fuerte con-

22 MacDonald, J. S. y MacDonald, L. D.: "Chain Migration, Ethnic Neighborhood Formation and Social Networks", The Milbank Memorial Fund Quartely, núm. 1, 1964, págs. 82-97. (traducción personal)

23 En la historiografía inglesa, el primer emigrante de una comunidad recibe varias denominaciones: pionero (pioneer), líder (leader), primero-en-llegar (first comer), siempre indicando su categoría de primer eslabón de la cadena migratoria (chain migration).

24 Los estudios microhistóricos sobre comunidades italianas, principalmente, sugieren que la actividad de la cadena migratoria está en directa relación con el grado de afinidad por amistad o parentesco que existe entre los agentes que provocan la relación (familia, amigos, líderes vecinales).

25 Los conceptos de self-generating elements y feedback están ampliamente consolidados en la historigrafía al uso.

26 Algunos autores definen región migratoria no como un sistema migratorio en origen, sino como una colección de líneas migratorias entre lugares de origen y destino.

27 Véanse los trabajos de Rosario Márquez, especialmente las ilustrativas Historias de América: la emigración española en tinta y papel, Huelva, 1994. La autora aborda de nuevo el tema en otro libro, donde abarca a su vez otros aspectos de la emigración temprana en el siglo XIX, punto de partida de la emigración masiva de finales del siglo: La emigración española a América (1765-1824), Oviedo, 1995, págs. 255-264. 
centración de migrantes por lugar de origen. Se propicia así la formación de barrios étnicos, los grupos de presión étnicos, la segmentación del mercado laboral, los mercados étnicos,... Por consiguiente, y en una época en que "la idea de arrancar viejas raíces era todavía algo extraño y terrorífico para la mayor parte de las personas", ${ }^{28}$ las cadenas migratorias permitieron humanizar el fenómeno, privilegiando las relaciones interpersonales y creando un ambiente psicosocial más acogedor para el migrante..$^{29}$

Para comprender el papel de estos procesos migratorios a niveles masivos, hay que tener en cuenta otro elemento tan obvio como fundamental: el tiempo. El mecanismo "cadena migratoria" se manifestó de modo más evidente y funcional luego que se estableció un grupo numeroso de migrantes en América, que configuró la base de los primeros eslabones de las múltiples cadenas que irían desarrollándose con los años. Este lapso de tiempo fue el que se necesitó para que las expectativas de migrar llegasen a ser contagiadas a otros miembros de la comunidad de origen, y para que los pioneros, integrándose en el nuevo mercado laboral, pusiesen en marcha el proceso de estímulo y ayuda financiera. En una pequeña comunidad rural la transmisión pudo ocurrir muy rápidamente; pero en unidades geográficas mayores el proceso se muestra más lento, porque los eslabones son relativamente menos numerosos, esto es, menos densos en el espacio.

\section{Crítica y comprobación de un modelo de difusión}

A fines del siglo XIX, gracias a la variada acción de los mecanismos hasta aquí descritos, hubo una apreciable difusión de la idea de emigrar en Europa, con diferencias cronológicas regionales. Y de ello hay evidencias cualitativas. No es extraño que, como ya hemos indicado, se hablase de "fiebre de la emigración" ("fever of emigration"). Los andaluces no quedaron inmunes a la sensación de contagio de esa fiebre, incluso en provincias con escasa tradición emigratoria contemporánea como ponía de manifiesto el diario La Andalucía de Sevilla: "La emigracion [...] es, más bien, la moda que impera, y a la que es necesario rendir culto; por esta causa,

28 Hobsbawn, E.J.: La era del capitalismo, Barcelona, 1981, págs. 297-298.

29 Vázquez, A. y Estrada, B.: “ Causas de la emigración...”, pág. 236. Por estas razones, se ha dicho que, por ejemplo, para un irlandés emigrar a Nueva York o a Boston era como buscar trabajo en una ciudad irlandesa. 
quizás, es por lo que la misma, ha logrado, arrastrándose como serpiente, introducir al fin y al cabo su cabeza, en nuestra provincia". ${ }^{30}$

También en la provincia Cádiz, que conocía emigraciones más recientes, muchos se asombraban de la celeridad que tomaba el contagio de la idea de emigrar, que parecía imparable a pesar de las lúgubres cartas publicadas por los periódicos intentando desmitificar las expectativas de América: "Sabemos que hay en Jerez nuevas cartas procedentes de la República Argentina en las que se lanzan amargas quejas por el inicuo trato que allí reciben los infelices emigrantes procedentes de ésta y otras provincias [...].- Lo inexplicable del caso es que continúa la fiebre de la emigración en aumento [...]". ${ }^{31}$

Según apunta J. D. Gould, esta metáfora, que también era usada en otros países europeos, resulta bien apropiada, en tanto que contiene la "implícita sugerencia de que la difusión de la idea [de migrar] se realiza vía la transmisión entre individuos en íntimo contacto unos con otros", ${ }^{32}$ esto es, mediante la dinámica de los procesos migratorios que hoy llamamos cadenas migratorias.

A este respecto, adoptaremos la definición de "difusión" que da Gould: progreso a través del tiempo de las intensidades de emigración. El indicador de la intensidad emigratoria lo representa convenientemente la tasa de emigración bruta por 1.000 habitantes.

Además, según el modelo explicativo de Gould, que comprueba para los casos italiano, húngaro y portugués, este incremento de la intensidad emigratoria, por ejemplo, a escala regional no resulta de un incremento uniforme de la intensidad en todas las subdivisiones geográficas (provincias), sino de un incremento desproporcionadamente mayor en las áreas que han sido previamente menos afectadas, o no afectadas en absoluto, por la emigración. Estas nuevas provincias emigratorias llegan a "suplementar", no a sustituir, conforme pasa el tiempo, las anteriores fuentes de emigración. Cuando esto ocurre, como sucedió en 1900-14 en el sur y este de Europa, incluida Andalucía, se alcanza el momento de "saturación emigratoria" o máxima extensión de la idea de emigrar del ciclo migratorio definido. Este momento se refleja gráficamente en la cima formada por la serie agregada de curvas en "S" que la emigración europea describe a diferentes escalas de estudio.

30 La Andalucía, Sevilla, 30 de junio de 1889.

31 El Guadalete, Jerez, 21 de mayo de 1889.

32 Gould, J. D.: "European Inter-Continental...”, pág. 304. (traducción personal) 
FRANCISCO CONTRERAS PÉREZ

TABLA 2

EMIGRACIÓN EXTERIOR DE ANDALUCÍA (POR PROVINCIAS), 1885-1887, 1888-1890 Y 1911-1913

Tasa de emigración media anual ( 1000 hab.)

\begin{tabular}{lccc} 
Prov. / región & $1885-1887$ & $1888-1890$ & $1911-1913$ \\
\hline Almería & 23,60 & 23,89 & 37,39 \\
Cádiz & 2,84 & 10,42 & 9,73 \\
Córdoba & 0,14 & 0,30 & 0,61 \\
Granada & 0,98 & 3,93 & 7,37 \\
Huelva & 0,23 & 0,69 & 0,63 \\
Jaén & 0,25 & 0,24 & 1,09 \\
Málaga & 1,19 & 10,02 & 7,19 \\
Sevilla & 0,43 & 1,13 & 2,17 \\
Andalucía & $\mathbf{3 , 1 4}$ & $\mathbf{6 , 0 4}$ & $\mathbf{7 , 5 2}$ \\
Galicia & 5,21 & 9,03 & 28,33
\end{tabular}

Fuente: Véase nota 1. Elaboración personal.

En Andalucía, las intensidades emigratorias registradas en los primeros años para los que hay estadísticas, y el ápice de la emigración exterior, se reflejan en la tabla 2. Observamos, en efecto, que este indicador de la emigración desde Andalucía y desde todas sus provincias se incrementó entre una fecha y otra. Este incremento de las intensidades emigratorias provinciales caracterizó la explosión emigratoria en Andalucía de 1911-13, esto es, el mencionado momento de "saturación" emigratoria.

Dicha evolución se refleja gráficamente en una serie de curvas de Lorenz, que expresan los porcentajes acumulados de las poblaciones totales y de la emigración total de las ocho provincias andaluzas (véase la fig. 4). Las curvas coincidentes con la diagonal de $45^{\circ}$, o sea la línea de equidistribución, indicarían que las provincias respectivas suministraron emigrantes al conjunto de la corriente regional en exacta proporción a su participación en el total poblacional. Y viceversa: a mayor amplitud del área entre la curva real y la diagonal de $45^{\circ}$, menor distribución de las intensidades de emigración.

La segunda parte del modelo de Gould indica que este incremento de la intensidad emigratoria regional se debió a un crecimiento relativamente 
mayor en las provincias de nueva emigración. En Andalucía, las tasas de variación relativa de las intensidades de emigración provinciales entre 188587 y 1911-13, confirman el mayor incremento relativo de las intensidades emigratorias en provincias con menor tradición migratoria que Almería. Efectivamente, las tasas de variación relativa de las otras siete provincias llegaron a estar entre el $87 \%$ de Granada y el $63 \%$ de Huelva. En menor proporción relativa aumentó la intensidad emigratoria en Almería (37\%), la provin-

FIG. 4.-CURVAS DE LORENZ DE LA EMIGRACIÓN EXTERIOR. ANDALUCÍA (porcentajes acumulados por provincias), 1885-1887, 1888-1890 Y 1911-1913

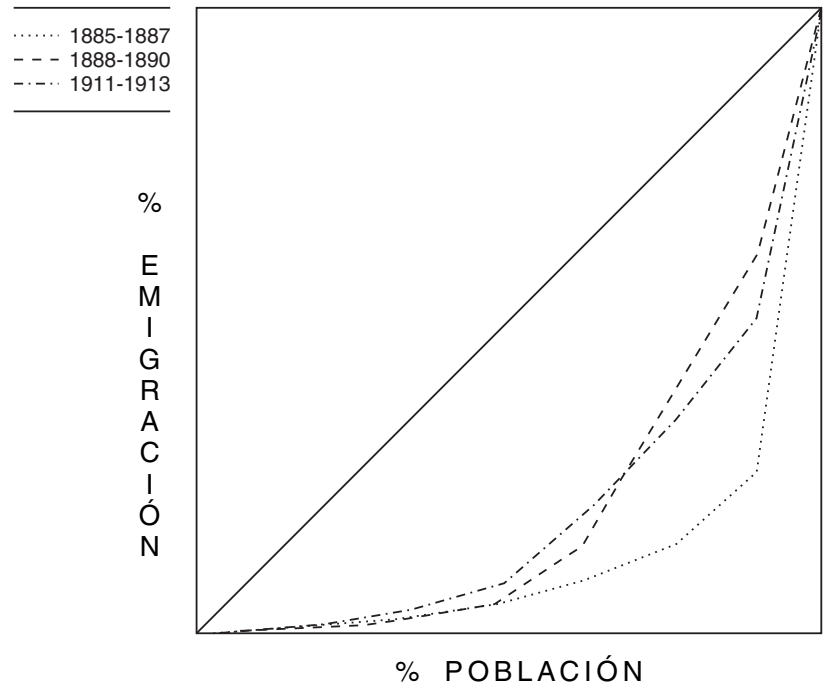

Fuente: Véase nota 1. Elaboración personal.

cia andaluza con la más poderosa y constante corriente emigratoria a fines del siglo XIX. Así pues, el incremento de la intensidad emigratoria andaluza se debió a la acción suplementaria del incremento relativo en las provincias de "nueva emigración", que eran la mayoría en Andalucía al cambiar el siglo.

Por consiguiente, desde 1885-87 se da en Andalucía un proceso de difusión de las expectativas emigratorias que, tras una fase de "maduración" (1885-1887), culmina con una etapa de "saturación" en 1911-13. En este último período, Andalucía alcanza su mayor tasa emigratoria (7,52 por 
1000), gracias a un incremento relativo de las provincias que menor intensidad emigratoria habían presentando a principios del período observado. Ahora bien, recordemos que, a pesar de ello, aumentaron las diferencias entre Andalucía y Galicia a este respecto, prueba de que no hubo en las corrientes americanas principalmente un proceso de realimentación del flujo emigratorio de similares proporciones.

En todo caso, sobre esta razonable coincidencia en la evolución de la divulgación de la idea de emigrar en Andalucía con respecto al modelo explicativo de Gould, debemos hacer algunas precisiones. En concreto, nos referimos a la primera oleada emigratoria andaluza de 1888-90 (véase la tabla 2), que adelantó de manera masiva el proceso que culminaría en 1911-13. Mientras la provincia con más tradición emigratoria, Almería, permaneció en una situación estable entre 1885-87 y 1888-90, Málaga y Cádiz asistieron a una especie de aceleración del proceso de difusión de las ansias emigratorias (a América en lugar del norte de África como los almerienses de por entonces). Tanto es así que, en comparación con las mismas tasas del período 1911-13, estas dos últimas provincias muestran valores superiores. En términos de distribución regional, el resultado se expresa en la curva de Lorenz (fig. 4). Se observa cómo los procesos migratorios desencadenados en 188890 dieron lugar a una aceleración de la transmisión de la idea de emigrar: la curva muestra una distribución ligeramente más equitativa que la que veremos en 1911-13. Esto es, en sólo tres años el ritmo de la difusión de la emigración permite adelantar la situación dibujada con posterioridad por la curva de 1911-13, años que hemos denominado de "saturación emigratoria".

Teniendo en cuenta las limitaciones de comunicación de la época y las reducidas dimensiones de los contingentes anteriores, esta aceleración en la propagación provincial de la "fiebre migratoria" sólo es explicable teniendo en cuenta mecanismos extraños a las cadenas migratorias, pues queda implícito que fueron desarrollados en un lapso de tiempo más reducido que el que necesitan las redes sociales para asentarse con efectividad y dimensión adecuadas. ${ }^{33}$ Por consiguiente, la explicación más plausible apunta al

33 Gould indica que la "difusión refleja el hecho que se requirió algún lapso de tiempo para que la idea de emigración llegase a implantarse en las mentes de los miembros de una comunidad, para que su beneficio neto fuera firmemente demostrado por la experiencia de los que han partido primero, y para que estos pioneros pusieran en marcha el proceso de estímulo y ayuda financiera que forman los eslabones constituyentes de la llamada 'cadena migratoria'. En una pequeña comunidad rural la 'difusión’ podría ocurrir muy rápidamente,...; pero entre comunidades [como sucede a nivel provincial] [...], el proceso fue más lento porque los eslabones eran relativamente menos numerosos". Gould, J. D.: "European Inter-Continental...", pág. 292-293 (traducción personal). 
papel de los agentes oficiales de emigración, cuya labor permite potenciar el ritmo de propagación de la idea de emigrar y en tramos temporales más reducidos (un año, por ejemplo). Si hasta entonces Almería poseía una potente corriente, más antigua y aislada en gran parte del resto de Andalucía, hacia Argelia, los nuevas redes de agentes americanos incidirán en otras provincias andaluzas de preferencia, de lo que resulta una mayor equidistribución provincial de la emigración exterior en sólo un año, 1889.

Ahora bien, si atendemos a la distribución de los planes de emigrar a América, como segmento particular de la emigración exterior andaluza, observamos igualmente una fuerte concentración. Pues, en realidad, los agentes concesionarios de los pasajes americanos llevaron a cabo su acción de manera bastante concentrada dentro de la región, en tanto que en un principio no debieron extender demasiado sus redes de captación para encontrar numerosos individuos dispuestos a emigrar, pero que hasta entonces habían carecido de la información sobre el destino y de la ayuda financiera suficiente para efectuar el viaje. Así pues, las localidades cercanas a los puertos de Cádiz, Gibraltar y Málaga, puertos donde las casas consignatarias de las navieras tenían sus sedes, fueron con mayor intensidad las más afectadas por estas reclutas.

La intensidad emigratoria en Málaga y Cádiz descenderá con posterioridad. De hecho, estas dos provincias presentan en 1888-90 indicadores emigratorios superiores a Galicia, pero hacia 1911-13 ya aparecen distanciadas en considerable medida de los valores presentados por este referente emigratorio por excelencia.

\section{Un análisis comparativo en un contexto mediterráneo}

Estimo que esta evolución de la distribución de la idea de emigrar no debe sugerir que se había alcanzado un hipotético nivel de saturación emigratoria en fecha tan temprana como 1888-90 (al menos temprana dentro de la tradición de emigración en masa al exterior), pues ni siquiera Galicia lo hace a pesar de tener una potente tradición emigratoria desde principios del siglo XIX y haber recibido también parte de los pasajes subsidiados argentinos.

A la luz de estos datos, lo que resulta bastante probable en el caso andaluz es que, como hemos observado en capítulos anteriores, la voluminosa emigración de 1888-90 no garantizó posteriormente una corriente de la dimensión relativa de la gallega. 
Si englobamos Andalucía en el conjunto de las regiones meridionales de Europa, podemos establecer su comparación respecto al caso italiano. La evolución de las intensidades de emigración en Italia se refleja en la tabla 3, distinguiendo las agrupaciones regionales. Al principio del ciclo emigratorio considerado, el conjunto de Andalucía parte con una tasa de emigración media anual similar a las del centro y sur de Italia.

TABLA 3

EMIGRACIÓN EXTERIOR DE ITALIA (POR REGIONES), 1881-1882 Y 1910-1912

Tasas de emigración media anual ( 1000 hab.)

\begin{tabular}{lcc} 
Región & $1881-1882$ & $1910-1912$ \\
\hline Norte & 8,3 & 17,3 \\
Centro & 2,2 & 15,4 \\
Sur & 4,1 & 21,1 \\
Italia insular & 0,6 & 19,5
\end{tabular}

Fuente: Gould: "European Inter-Continental...”, pág. 284.

El sur de Italia pasaba hacia esos años por procesos económicos similares a los vividos en Andalucía. Se estaban produciendo importantes cambios en la tenencia y el aprovechamiento de la tierra, que trajeron consigo el reforzamiento del latifundismo, el absentismo y la crisis del pequeño propietario. En suma, se estaban reforzando las desigualdades económicas y sociales regionales, en perjuicio del ámbito agrario dentro del proceso de modernización del país. ${ }^{34}$

El recurso a la emigración de Italia fue difundiéndose en el centro y sur del país, como en Andalucía a lo largo del tiempo (véase la fig. 5). Pero esta difusión no fue igual en las tres regiones. Mientras Andalucía no llega a superar el techo del 8 por 1000 en su ápice emigratorio, por entonces las dos regiones italianas ya habían duplicado este valor. Así pues, como adelantamos a la luz de la comparación con el caso gallego, observamos de nuevo la debilidad relativa de la corriente transoceánica andaluza, la existencia de obstáculos internos a los procesos migratorios, que impedía su desarrollo a pesar de sumarse en el siglo XX otros determinantes objetivos.

34 Foerster, R. F.: The Italian Emigration of our Times, Londres, 1924, págs. 64-82. 
FIG. 5.-CURVAS DE LORENZ DE LA EMIGRACIÓN EXTERIOR. ITALIA CENTRAL (A) Y MERIDIONAL (B)

(porcentajes acumulados por provincias), 1881-1882, 1900-1901 Y 1910-1912
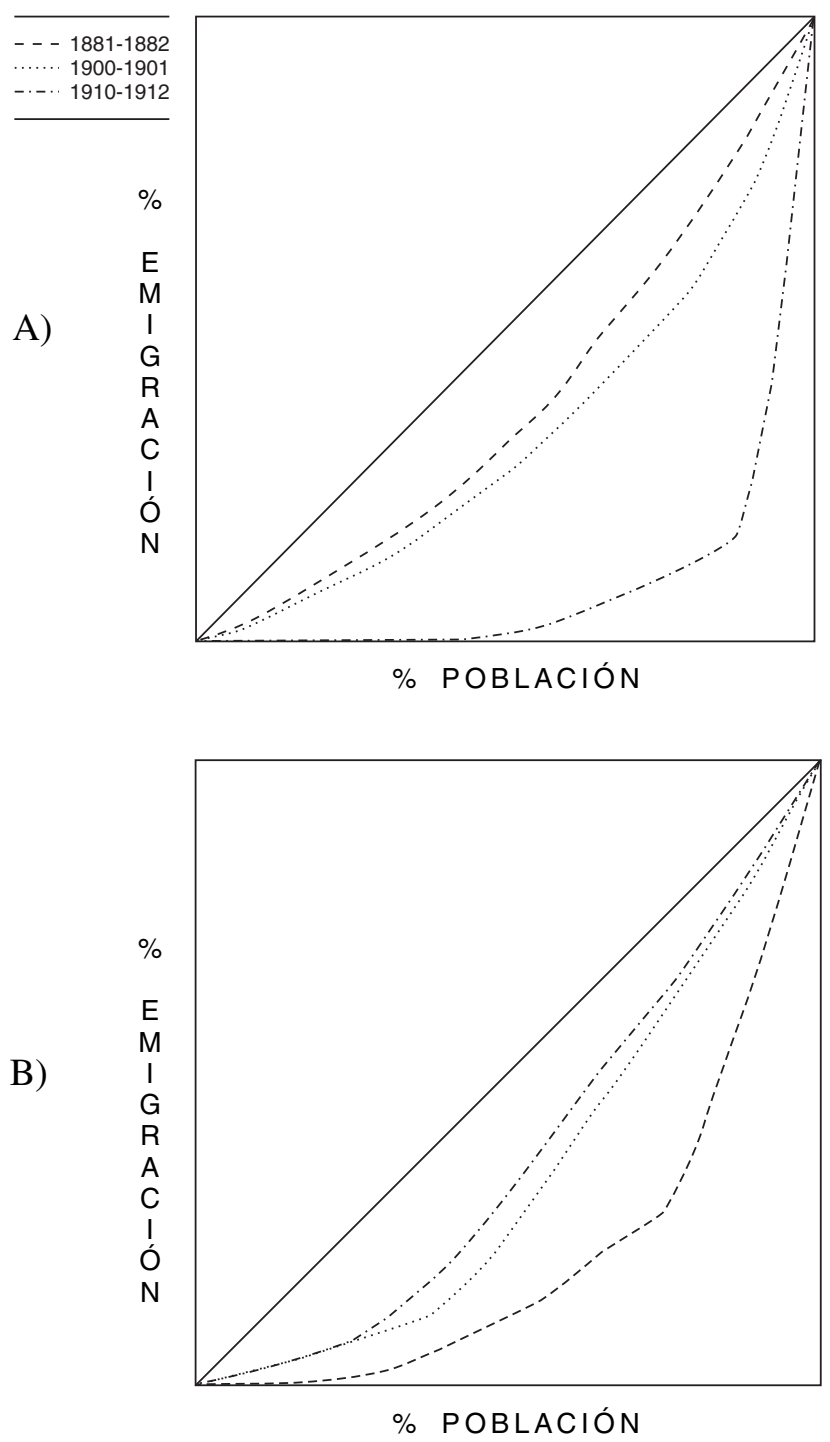

Fuente: Gould: “European Inter-Continental...”, págs. 284-285. 


\section{Conclusiones}

Como tendencia de la emigración en masa en Andalucía, desde 188587 se da un proceso de difusión de la idea de emigrar que, tras una fase de "maduración", culmina con una etapa de "saturación" en 1911-13. En este último período la región andaluza alcanza su mayor tasa emigratoria, gracias a un importante incremento relativo de la intensidad del fenómeno en las provincias con menor tradición en el mismo. El ritmo de esta divulgación no fue uniforme a lo largo del tiempo, produciéndose aceleraciones coyunturales, por la incidencia de importantes mecanismos posibilitadores de la emigración ajenos a las propias cadenas migratorias.

Aunque el uso de los agregados provinciales plantea obvias limitaciones como fuente para el estudio de la difusión de las expectativas emigratorias a nivel intercomarcal, es de reconocer que permiten una primera aproximación a la misma (se nos antoja una comparación con la dilatación de un zoom fotográfico). Ahora bien, su precisión depende de la configuración geográfica de las unidades provinciales que tratamos (extensión, facilidad de comunicaciones y acceso a puertos, concentración de la población,...). Es más, sabemos que los límites provinciales no dejan de ser convencionalismos administrativos, y por lo tanto no tienen por qué coincidir con las regiones migratorias, esto es, con las comarcas reales.

Teniendo en cuenta lo dicho, como hipótesis de trabajo parece razonable apuntar que las evidencias obtenidas nos llevan a afirmar que los emigrantes andaluces no asentaron redes sociales en destino tan potentes y definidas como las de gallegos, napolitanos y sicilianos. A falta de estos mecanismo posibilitadores de la emigración transoceánica, es muy probable que persistiera una importante emigración potencial o latente, no cuantificable, en Andalucía. A raíz de la acentuación de las desigualdades regionales originadas por los procesos de modernización, esta creciente emigración latente sólo llegaría a tener viabilidad efectiva, si adoptamos una perspectiva secular y colmatándose las posibilidades de los ámbitos urbanos andaluces, en la década de 1960 a propósito del éxodo continental hacia Cataluña y Europa $^{35}$. Había un acervo migratorio sólido, entendiendo tal como un conjunto de estrategias y hábitos culturales favorables a emigrar.

35 García Fernández, J.: La emigración exterior de España, Barcelona, 1965, págs. 136-289. 